\title{
Properties of nearby clusters of galaxies
}

\section{A 76, A 157, A 407, A 505, A 671, A 779, A 1700, A 2028, A 2040, A 2052, A 2063, A 2065, A 2593, A 2657, A 2670^}

\author{
D. Trèvese ${ }^{1}$, G. Cirimele ${ }^{1}$, A. Cenci ${ }^{1}$, B. Appodia ${ }^{1}$, P. Flin ${ }^{2}$, and P. Hickson ${ }^{3}$ \\ 1 Istituto Astronomico, Università di Roma "La Sapienza", via G. M. Lancisi 29, I-00161 Roma, Italy \\ 2 Institute of Physics, Pedagogical University, Ul. Lesna 16, 25-509 Kielce, Poland \\ 3 Department of Geophysics and Astronomy, University of British Columbia, 2219 Main Mall, Vancouver, BC, V6T 1Z4, Canada
}

Received October 30, 1996; accepted January 29, 1997

\begin{abstract}
We present $F$ band photometry, from digitized 48-inch Palomar plates, of 2818 galaxies brighter than $m_{3}+3$ in 15 Abell clusters. For each galaxy, absolute coordinates, magnitude, size, ellipticity and orientation are given. For each cluster we provide finding charts and contour maps of the galaxy surface density. The absolute coordinates of the galaxies of other 8 clusters presented in the first paper of this series are also included.
\end{abstract}

Key words: galaxies: clusters of: general

\section{Introduction}

We have undertaken a uniform analysis of a large sample of galaxy clusters to allow statistical studies of the clusters properties (Flin et al. 1988). This is the third of a series of papers (Trèvese et al. 1992 (T92); Flin et al. 1995) giving the positions, magnitudes, ellipticities and orientations of cluster galaxies having magnitudes between $m_{3}$ and $m_{3}+3$, where $m_{3}$ is the magnitude of the third brightest galaxy within one Abell radius of the cluster center, together with finding charts and cluster surface density maps for 2818 galaxies in 15 Abell clusters. Details of the observations and data reduction are described in T92. Thus in Sect. 2 only the main features of the data and the reduction procedures are briefly described. Since in the first paper of this series only relative galaxies positions were given, we include in the present paper their absolute coordinates.

Send offprint requests to: D. Trèvese

* Tables 3 to 17 of the present paper and Tables 18 to 25 (coordinates of the galaxies of Paper I) are available only in electronic form at the CDS via anonymous ftp to cdsarc.u-strasbg.fr (130.79.128.5) or via http://cdsweb.ustrasbg.fr/Abstract.html

\section{Observational data and reduction procedures}

Our data are derived from 10-inch photographic plates taken with the 48-inch Palomar Schmidt Telescope (Hickson 1977). The emulsions employed were Kodak 12702 or Kodak 098-04, both used with $2 \mathrm{~mm}$ Schott RG-1 glass filter, which corresponds to the red photographic $F$ band of Oemler (1974). Plates were calibrated using the Palomar spot sensitometer.

Fields containing the clusters originally selected by Hickson (1977), as well as some additional Abell clusters well visible on the plates, were scanned in transparency mode using a PDS 1010G micro-densitometer in Rome, producing a digital image for each cluster field having pixel sizes ranging from 10 to $25 \mu \mathrm{m}$, according to the noise level of the plate and cluster distance. The signal to noise ratio is $S / N \gtrsim 100$ for a few objects brighter than $12 \mathrm{mag}$, about 25 for $F \approx 14 \mathrm{mag}$ and falls to about 5 for $F \gtrsim 18$ mag. Objects are automatically detected and magnitude values are computed in many circular apertures, thus producing a magnitude profile from which objects are automatically classified as point-like or diffuse.

Total magnitudes $m_{\mathrm{T}}$ are computed from the flux integrated in an aperture whose radius is $R_{1}=1.5 r_{1}$, where $r_{1}$ is the first momentum of the intensity distribution (see T92).

With the above definition, total magnitudes correspond on average to the magnitude $m_{\text {iso }}$ computed in a circular aperture determined by the isophote $\mu=24 \mathrm{mag}$ $\operatorname{arcsec}^{-2}$, with the advantage that $r_{1}$ is less noisy than the corresponding isophotal radius (see Flin et al. 1995). Magnitude zero points are taken from the literature as indicated in Sect. 3. For comparison we give also the magnitude computed in a fixed aperture of 5 pixels radius. The corresponding value of the radius in arcsec is given in Table 1 for each cluster. 
The ellipticity and the orientation of each object are computed from the second-order momenta of the intensity distribution.

In each field a reference set of about 10 (properly distributed) stars belonging to the Guide Star Catalog of STScI (GSC), has been selected. Then, the right ascension $\alpha$ and declination $\delta$ referred to 2000 have been computed from the rectangular scanning coordinates, using the COORDS utility from the IRAF package. The resulting $\alpha$ and $\delta$ show a rms deviation smaller than 2 arcsec from GSC coordinates.

Density images of the clusters have been computed with a different method with respect to the two previous papers. The new algorithm applies an adaptive filter which operates a smoothing of the number density distribution using a Gaussian kernel with density dependent variance, as described in Pisani (1993), see also Merritt \& Tremaine (1994). Density images were computed using all galaxies brighter than $m_{3}+2$ for the clusters A76, A157, A2028, A2052 and A2657 while for the remaining 10 clusters we adopted $m_{3}+3$ as limiting magnitude. Isodensity maps of each cluster field are shown in Figs. $1 \mathrm{~b}$ to $15 \mathrm{~b}$

For comparison we have computed also the isodensity images of each cluster with the algorithm adopted in Papers I and II. It appears that the new method provides smoother isopleths without loss of spatial resolution. As an example we show in Figs. 16 the isodensity map of the irregular cluster A407, computed with the algorithm of Papers I and II.

Table 1. Cluster data

\begin{tabular}{lccccl}
\hline Abell \# & $N$ & $z$ & $\alpha(1950)$ & $\delta(1950)$ & $\phi_{\mathrm{a}}\left({ }^{\prime}\right)$ \\
\hline A76 & 1 & 0.0416 & 003650 & +062700 & 8.4 \\
A157 & & $0.103^{\dagger}$ & & & 6.7 \\
A407 & 1 & 0.0463 & 025844 & +353823 & 6.7 \\
A505 & 1 & 0.0543 & 045112 & +800609 & 7.4 \\
A671 & 1 & 0.0502 & 082527 & +303602 & 7.4 \\
A779 & 1 & 0.0230 & 091646 & +335729 & 7.4 \\
A1700 & & $0.119^{\dagger}$ & & & 5.0 \\
A2028 & 1 & 0.0776 & 150701 & +074417 & 6.7 \\
A2040 & 3 & 0.0456 & 151020 & +073742 & 6.7 \\
A2052 & 1 & 0.0348 & 151412 & +071226 & 6.7 \\
A2063 & 1 & 0.0337 & 152039 & +084714 & 6.7 \\
A2065 & 18 & 0.0722 & 152018 & +275349 & 6.7 \\
A2593 & 1 & 0.0421 & & & 6.7 \\
A2657 & 1 & 0.0414 & 234225 & +085502 & 8.4 \\
A2670 & 1 & 0.0761 & 235140 & -104143 & 6.7 \\
\hline
\end{tabular}

$\dagger$ the redshift has been estimated from the $z-m_{10}$ relation

\section{Description of the catalog}

The clusters studied in this paper are listed in Table 1. The column headings are:

Column 1: Abell catalog number.

Column 2: Identification number of the galaxy used to set the zero of the magnitude scale.

Column 3: Redshift (from Struble \& Rood 1991).

Column 4: Right ascension of the galaxy in the reference paper.

Column 5: Declination of the galaxy in the reference paper.

Column 6: Radius adopted for fixed aperture photometry, in arcsec.

The zero of the magnitude scale has been established using published photometric data. The reference papers are listed below.

For A76, and A2657 we have used $r$ magnitudes of bright galaxies given by Hoessel et al. (1980), and compared our photometry in the same aperture after the transformation $F=r-0.58$, valid for bright elliptical galaxies, for which we assume $B-V=0.97$ (Schneider et al. 1983) (SGH). For A157 we used the zero of A151, which lies in the same plate (see Paper II). For the clusters A671, A779, A2028, A2040, A2052, A2063, A2065, and A2670 we have used the $r$ magnitudes of the brightest member taken from Hoessel \& Schneider (1985) after the transformation $F=r-0.58$, from SGH.

For A1700 (which lies in the same plate of A1661) and A2593 we have used the $V$ magnitudes of Bothun et al. (1985), Murphy et al. (1983) respectively, after the transformation $F=V-0.76$, from SGH. For A407 and A505 we used the $V$ magnitudes of Peterson (1970) after the transformation $F=V-0.76$. The error on the zero of the the magnitude scale can be deduced from the above references.

Each catalog contains all the galaxies in the area shown in the identification maps, brighter than $m_{3}+3$.

Data for the individual galaxies in each cluster are given in Tables 3 to 17 . The column headings for these tables are as follows:

Column 1: Identification number.

Column 2: Right ascension 2000.

Column 3: Declination 2000.

Column 4: Size in arcsec, defined in Eq. (1) of Paper I.

Column 5: Ellipticity $(1-b / a)$, where $b / a$ is the ratio of minor/major axis lengths.

Column 6: angle between the major axis and to the positive $y$ direction, in degrees, increasing counterclockwise.

Column 7: Total $F$ magnitude integrated in a circular area of radius $R_{1}$.

Column 8: $F$ magnitude in a fixed circular aperture, whose radius in arcsec is indicated in Table 1.

Galaxies are sorted according to their total magnitude. 
Fore each cluster we give, in Figs. 1 to 15, identification maps with the serial numbers of the first 50 objects and isodensity plots obtained from the density images.

Table 2 contains the data of 2 bright galaxies of A1213, missing in T92, in the same format of Tables 2-16.

Tables 18-25 contain the $\alpha$ and $\delta$ (2000) coordinates of the galaxies belonging to the clusters A195, A465, A1185, A1213, A1413, A1775, A2319, A2597, which were presented in T92 using relative coordinates.

Table 2. addendum to Trèvese et al. (1992): Abell 1213

\begin{tabular}{|c|c|c|c|c|c|c|}
\hline$\alpha$ & $\bar{\delta}$ & $R_{1}$ & $\epsilon$ & $\bar{\theta}$ & $m_{\mathrm{T}}$ & $m_{\mathrm{a}}$ \\
\hline 111627.1 & +291433 & 16 & 0.29 & 69 & 13.8 & 14.8 \\
\hline 111622.0 & +291304 & 16 & 0.44 & 143 & 13.8 & 14.0 \\
\hline
\end{tabular}

Acknowledgements. P.H. is supported by grants from the Natural Science and Engineering Research Council of Canada. P.F. thanks the Istituto Astronomico, Università di Roma "La Sapienza" for hospitality. This work has been partly supported by the Polish Committee for Scientific Research under the grant P304 006 05/93 and by the Italian MURST and CNR (G.N.A.).

\section{References}

Bothun D.G., Aaronson M., Schommer B., et al., 1985, ApJS 57,423

Flin P., Hickson P., Pittella G., 1988, in Large Scale Structures in the Universe. Observational and Analytical Methods, Seiter W.C., Duerbeck H.W., Tacke W. (eds.). Springer Verlag, p. 179

Flin P., Trévese D., Cirimele, Hickson P., 1995, A\&AS 110, 313

Hickson P., 1977, ApJ 217, 16

Hoessel J.G., Gunn J.E., Thuan T.X., 1980, ApJ 241, 486

Hoessel J.G., Schneider D.P., 1985, AJ 90, 1648

Merrit D., Tremaine S.D., 1994, AJ 108, 514

Murphy H.P., Schild R.E., Weekes T.C., 1983, MNRAS 202, 1127

Oemler A., 1974, ApJ 194, 1

Peterson B.A., 1970, AJ 75, 695

Pisani A., 1993, MNRAS 265, 706

Schneider D.P., Gunn J.E., Hoessel J.G., 1983, ApJ 264, 337 (SGH)

Struble F.S., Rood H.J., 1991, ApJS 77, 363

Trévese D., Flin P., Migliori L., Hickson P., Pittella G., 1992, A\&AS 94, 327 (T92) 


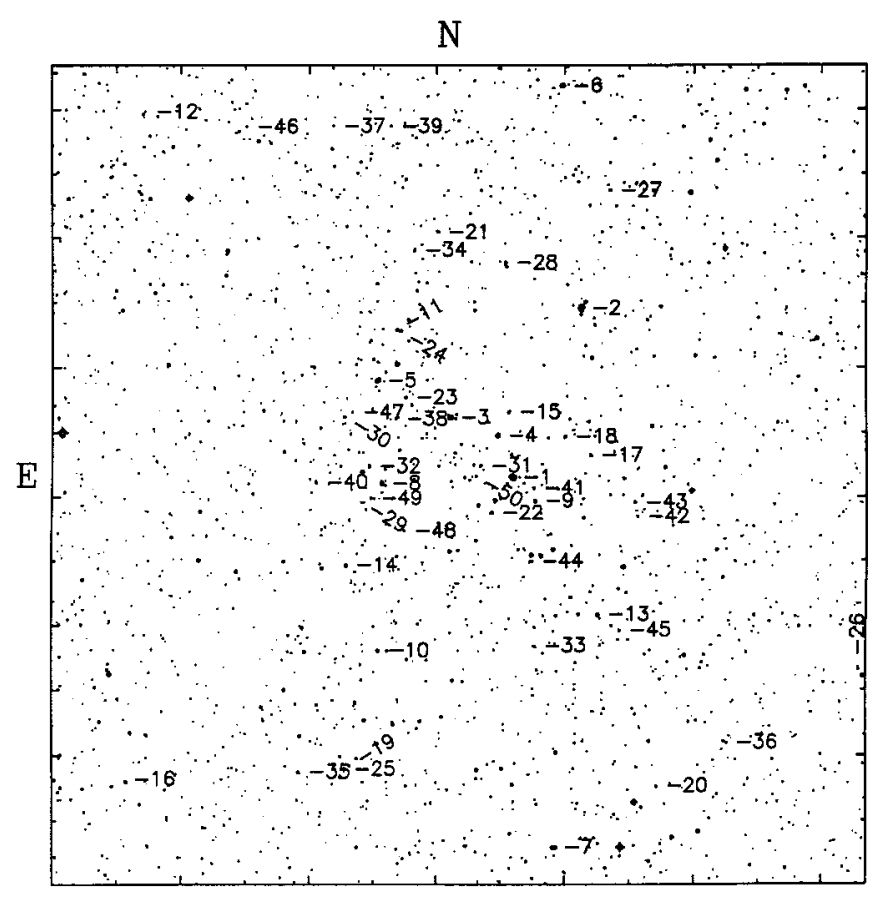

Abell 76

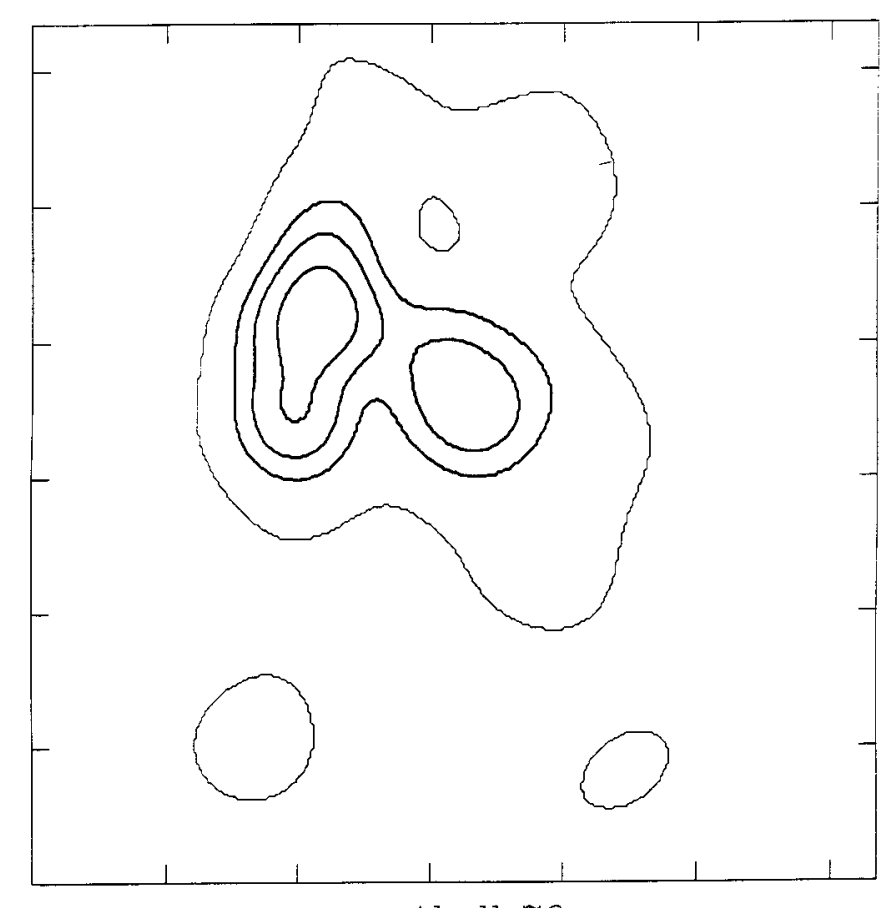

Abell 76

Fig. 1.

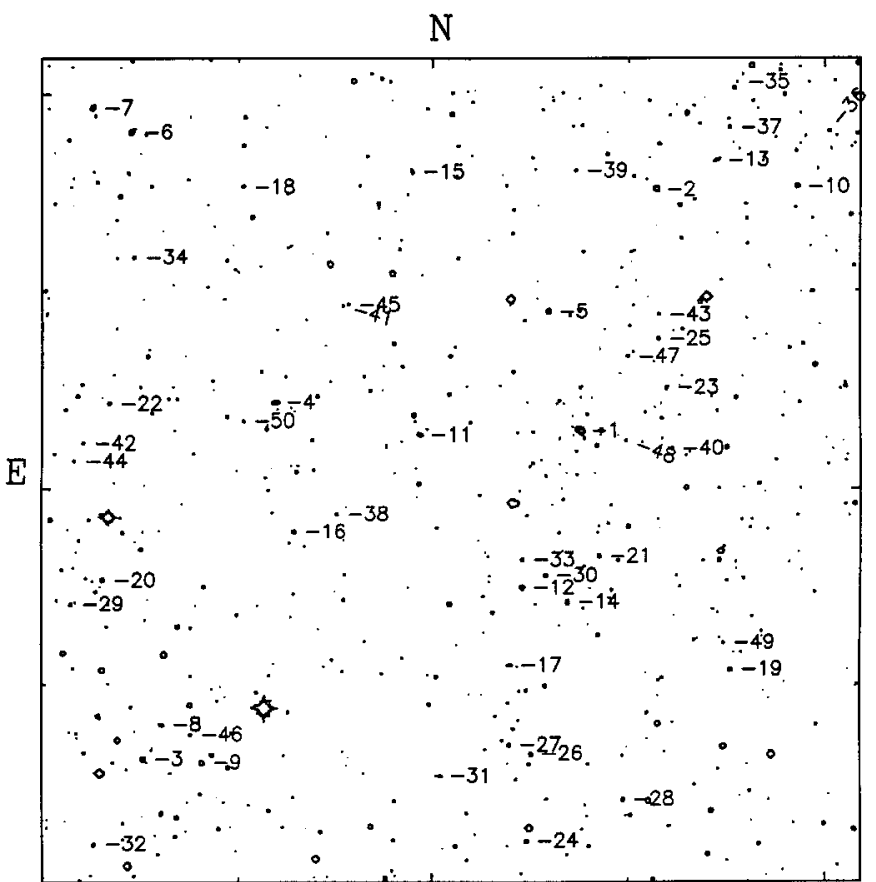

Abell 157

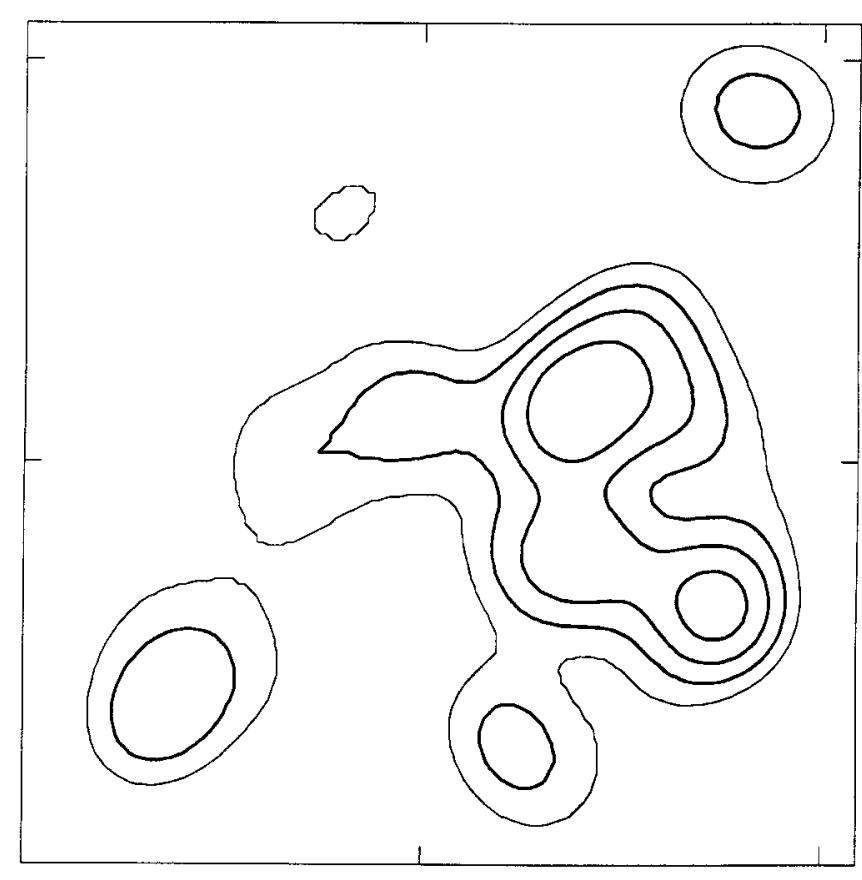

Abell 157

Fig. 2 .

Fig. 1 to 15. For each cluster field: a) identification maps with labels indicating the 50 brightest galaxies; b) isodensity maps, with the thick line indicating the average density of the field. The orientation is $\mathrm{N}$ at top, $\mathrm{E}$ at left. Big ticks are spaced by 15 arcmin. For each cluster the scale is the same in Figs. a) and b) 


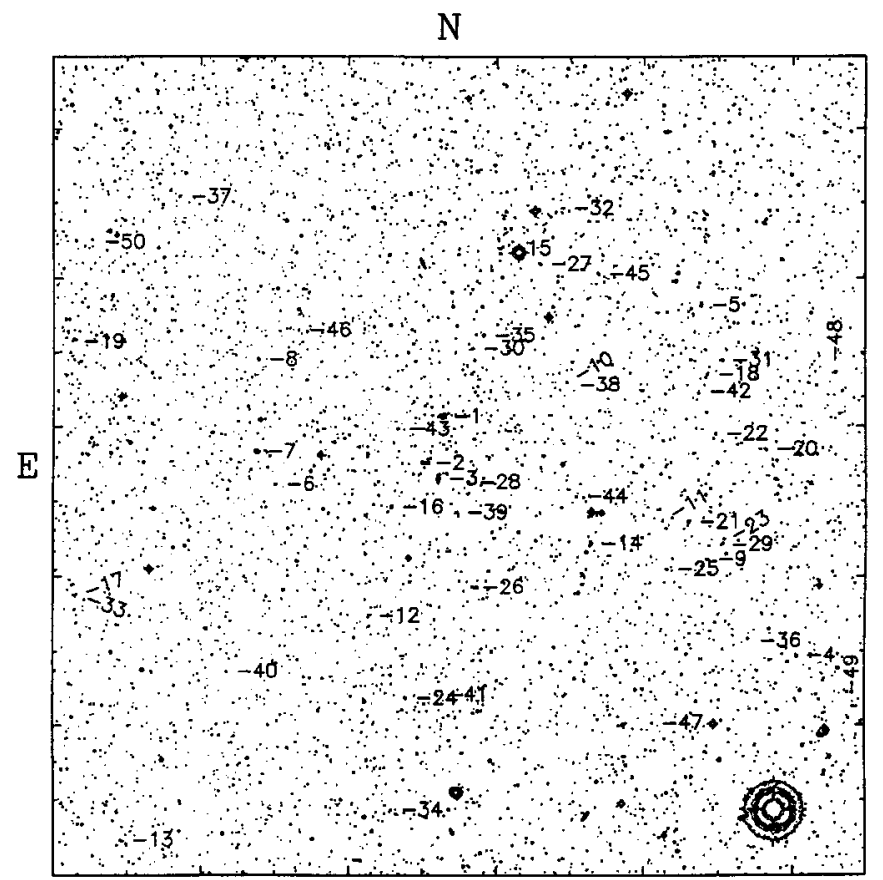

Abell 407

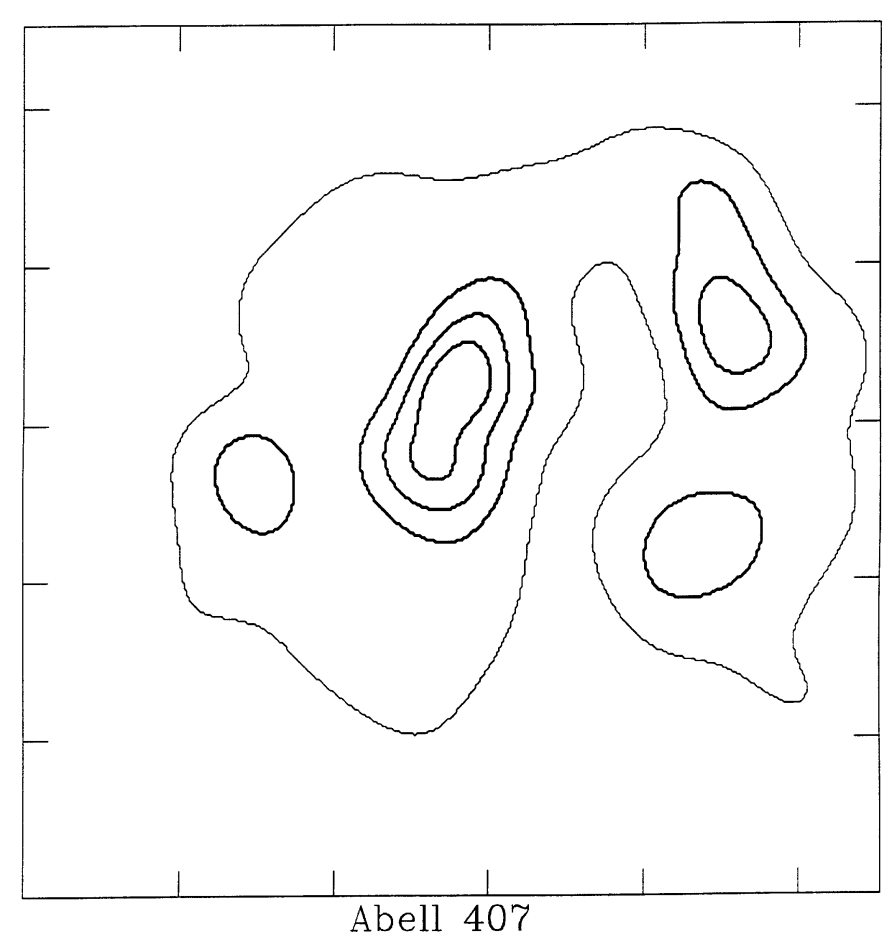

Fig. 3.

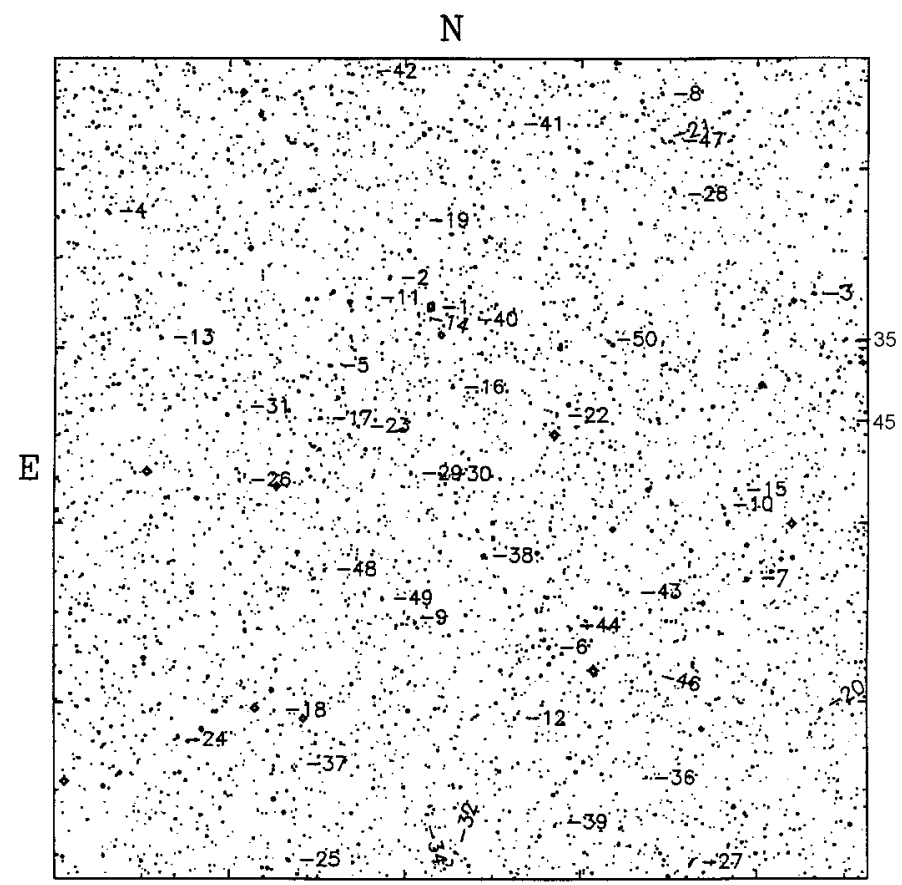

Abell 505

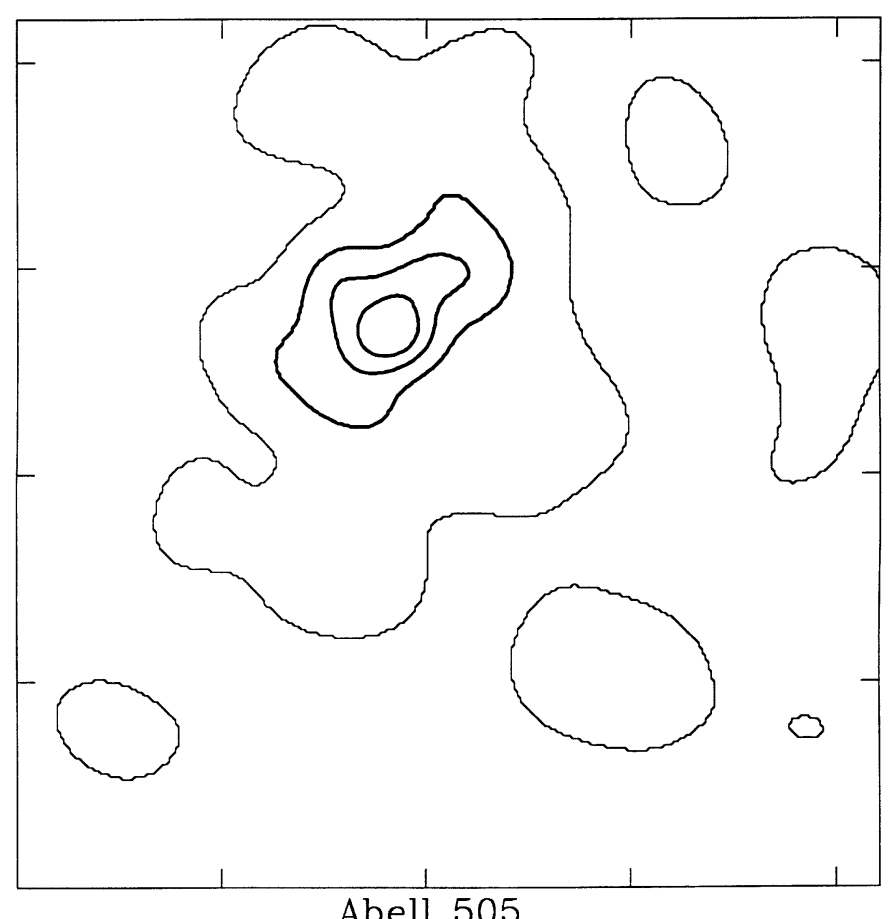

Abell 505

Fig. 4. 


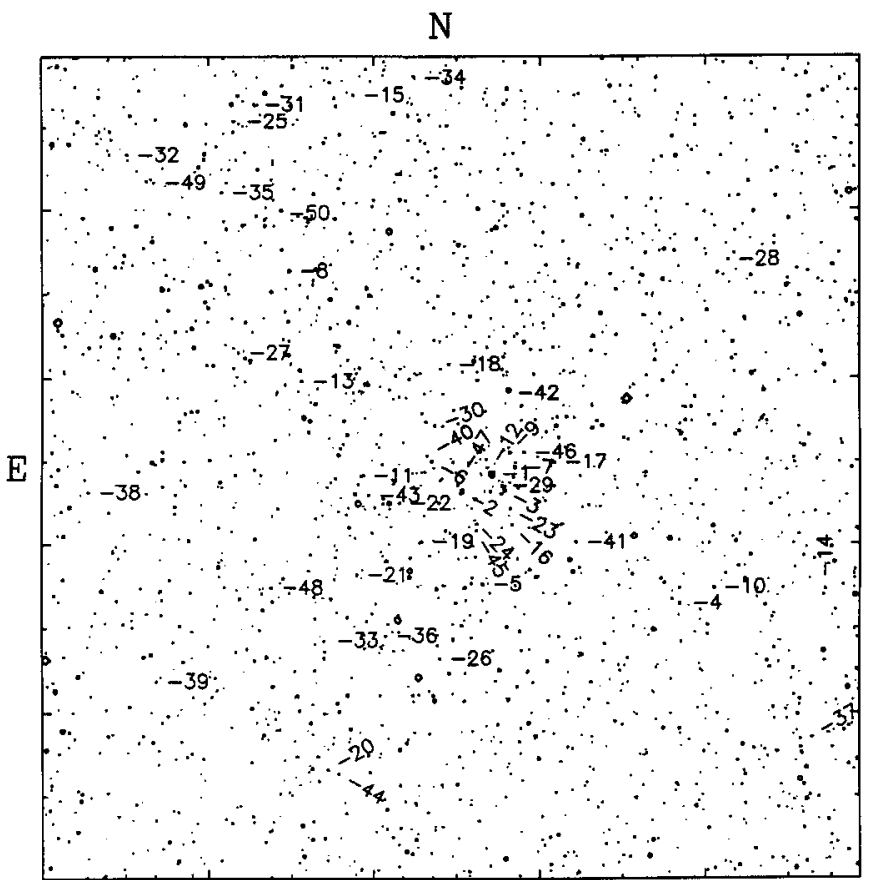

Abell 671

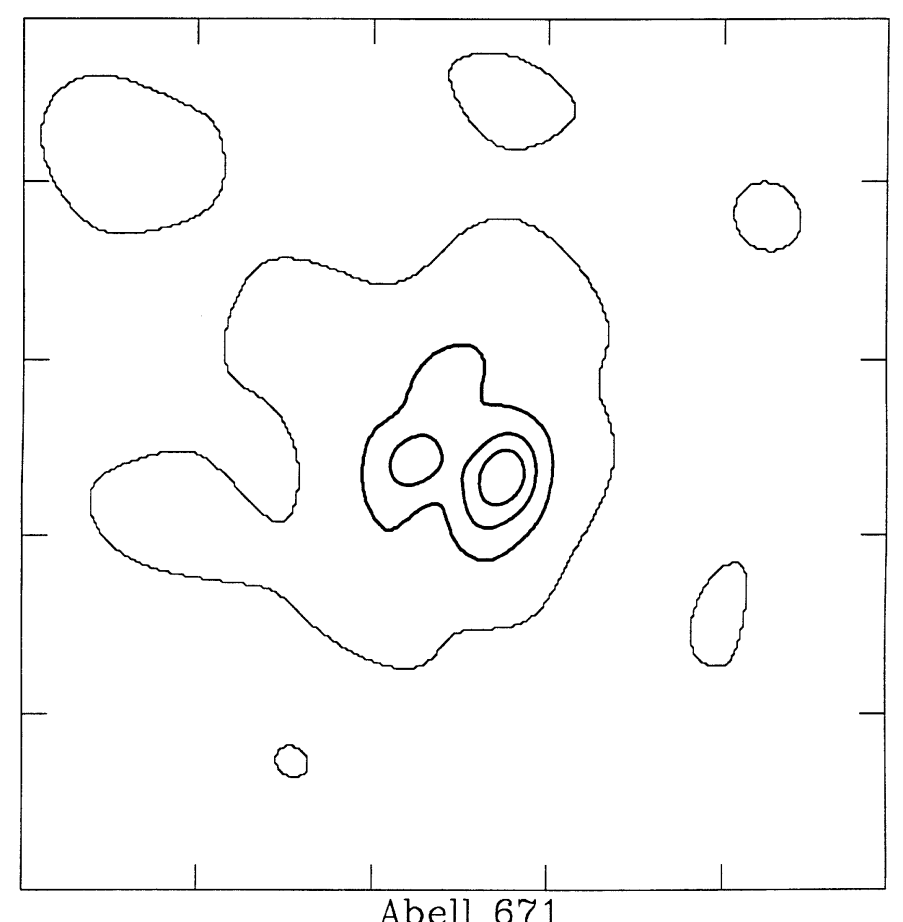

Abell 671

Fig. 5.

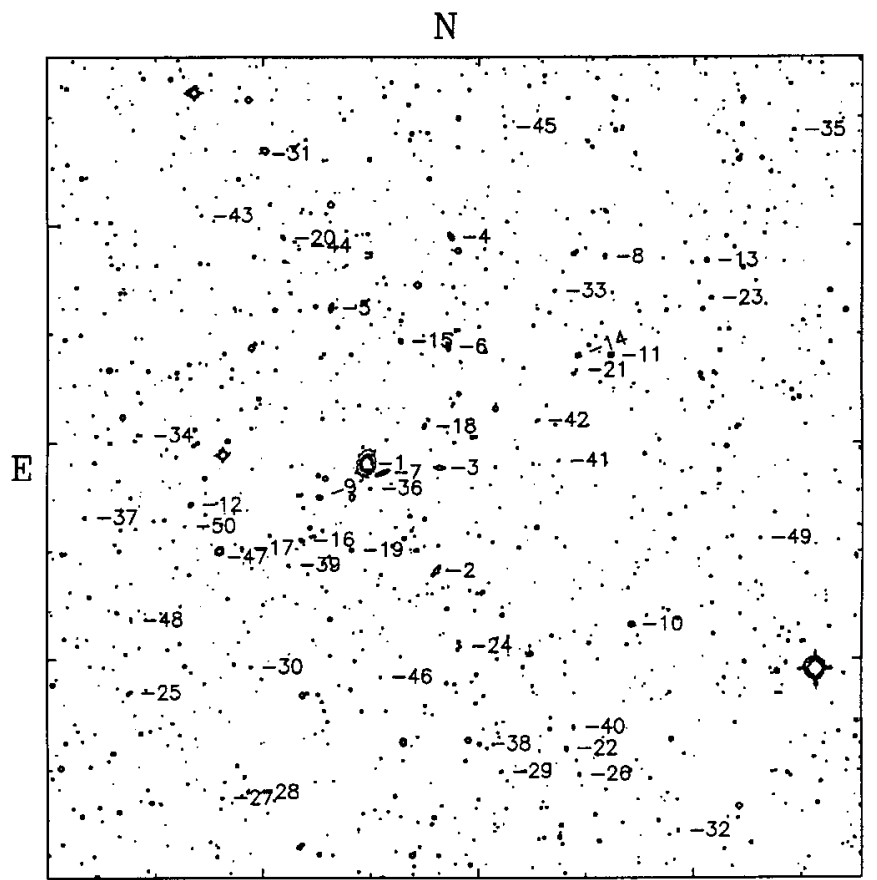

Abell 779

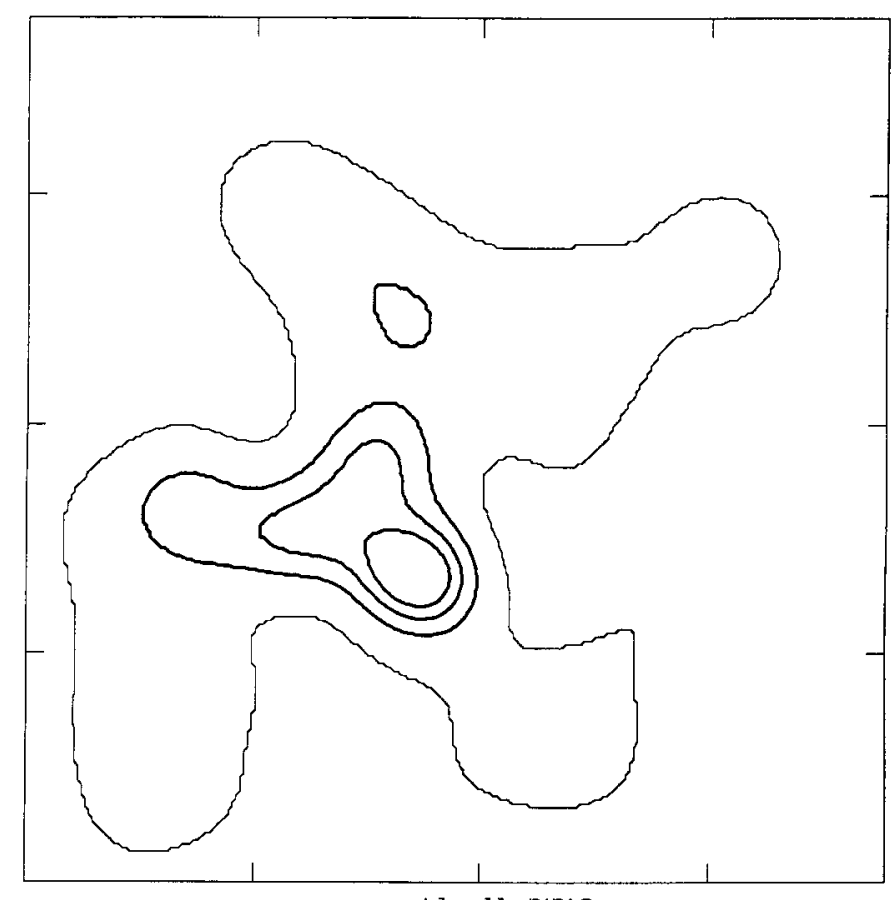

Abell 779

Fig. 6. 


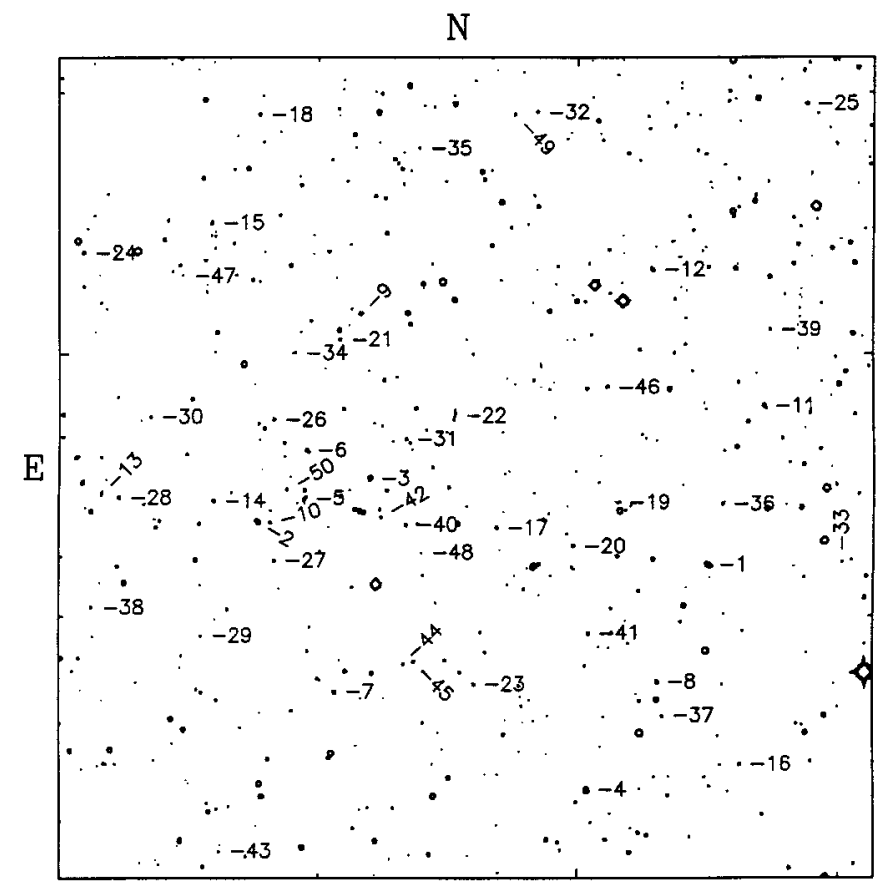

Abell 1700

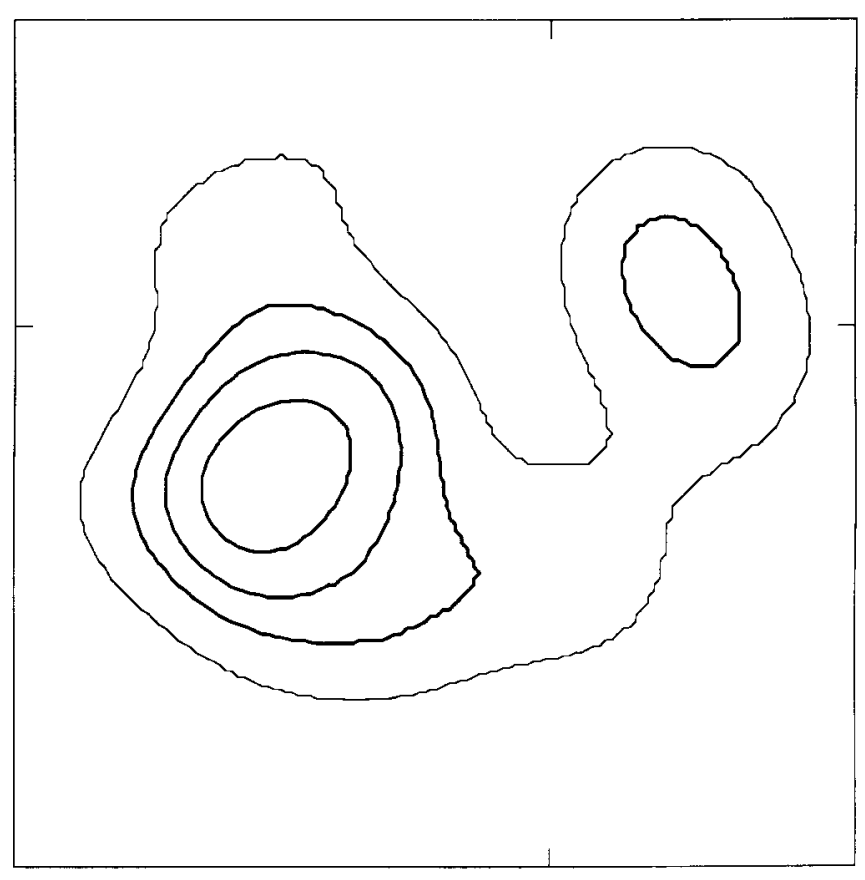

Abell 1700

Fig. 7 .

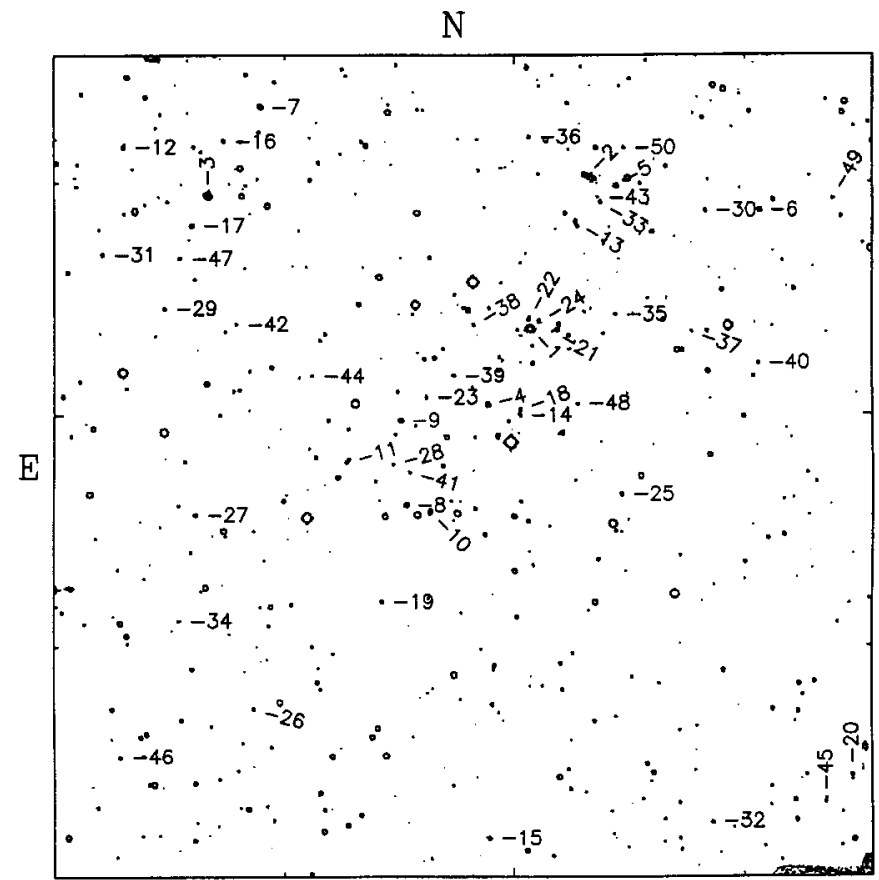

Abell 2028

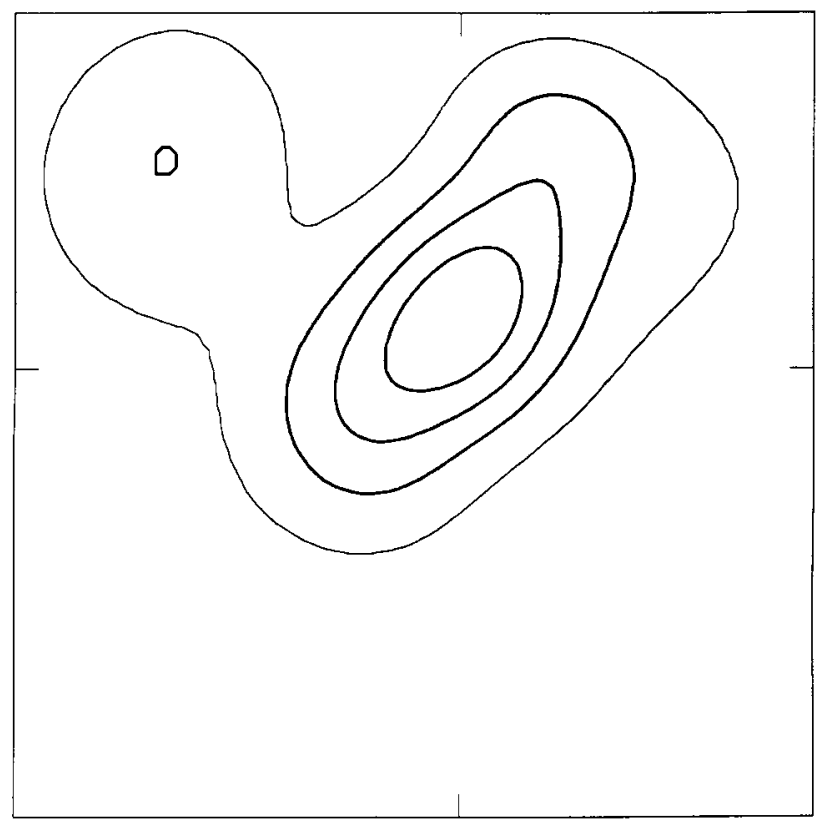

Abell 2028

Fig. 8 . 

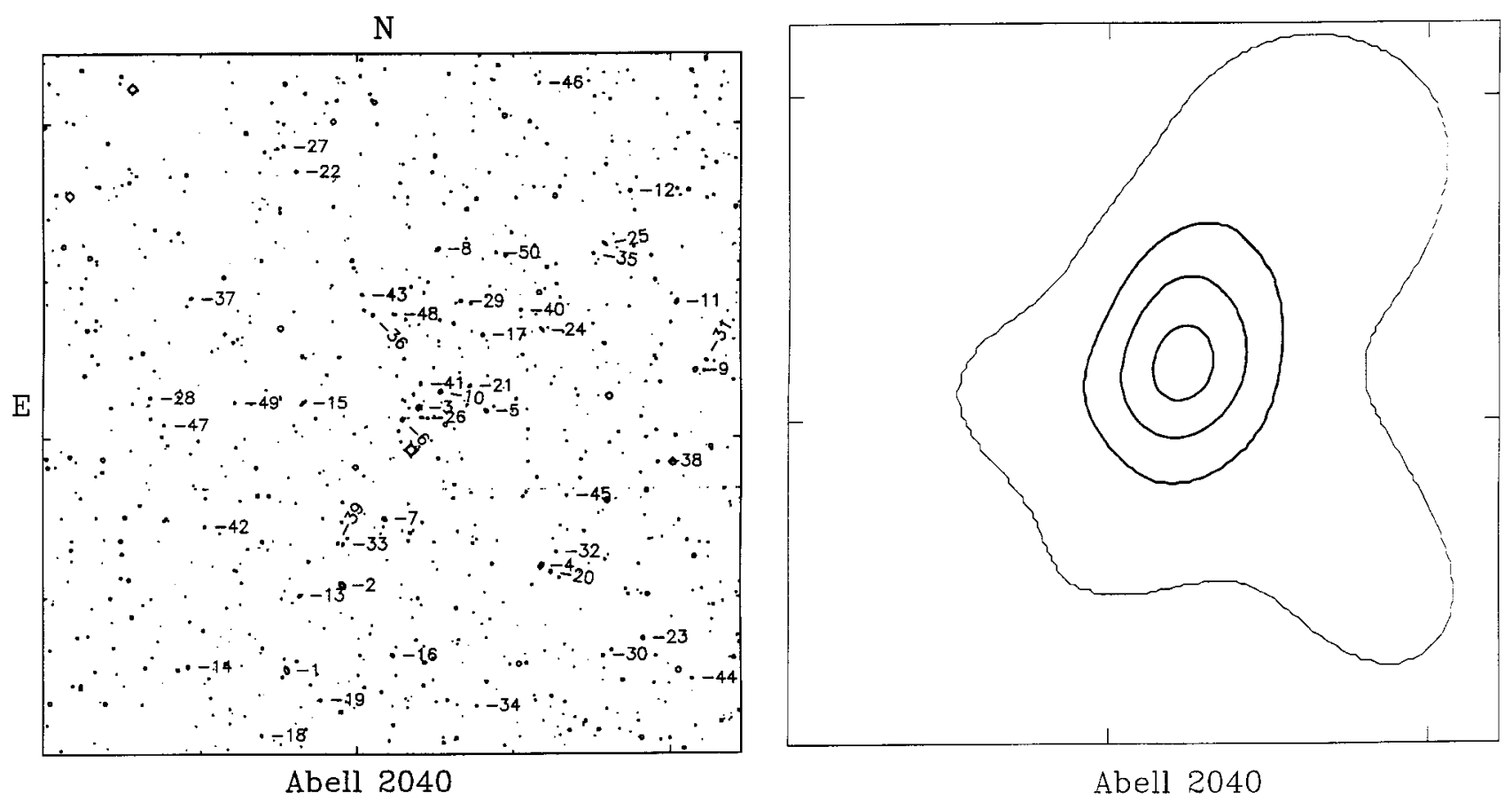

Fig. 9.

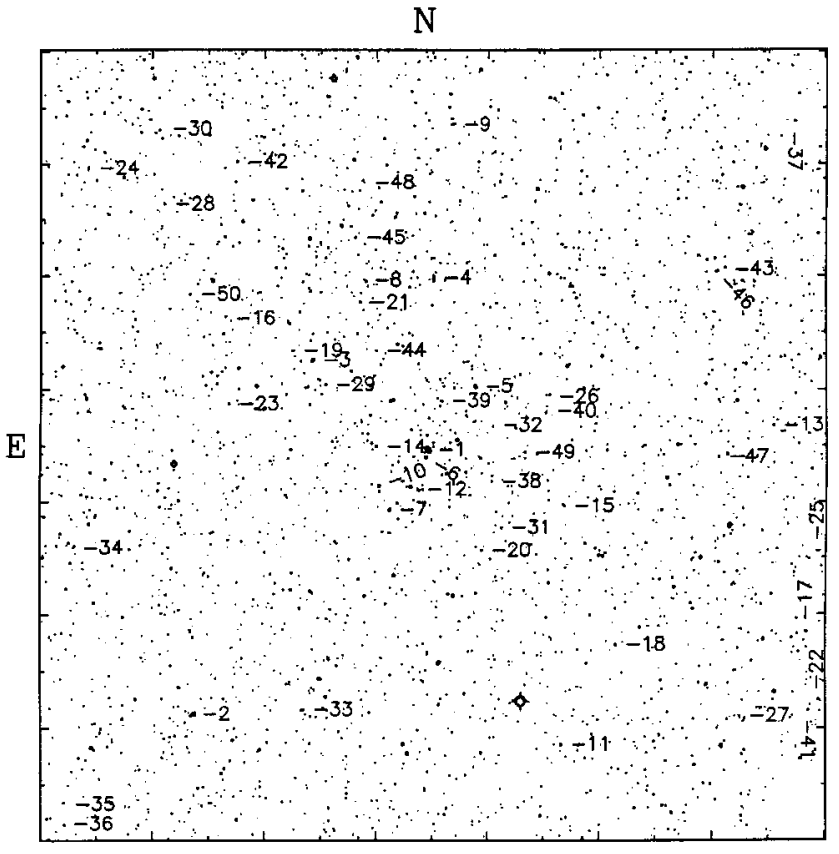

Abell 2052

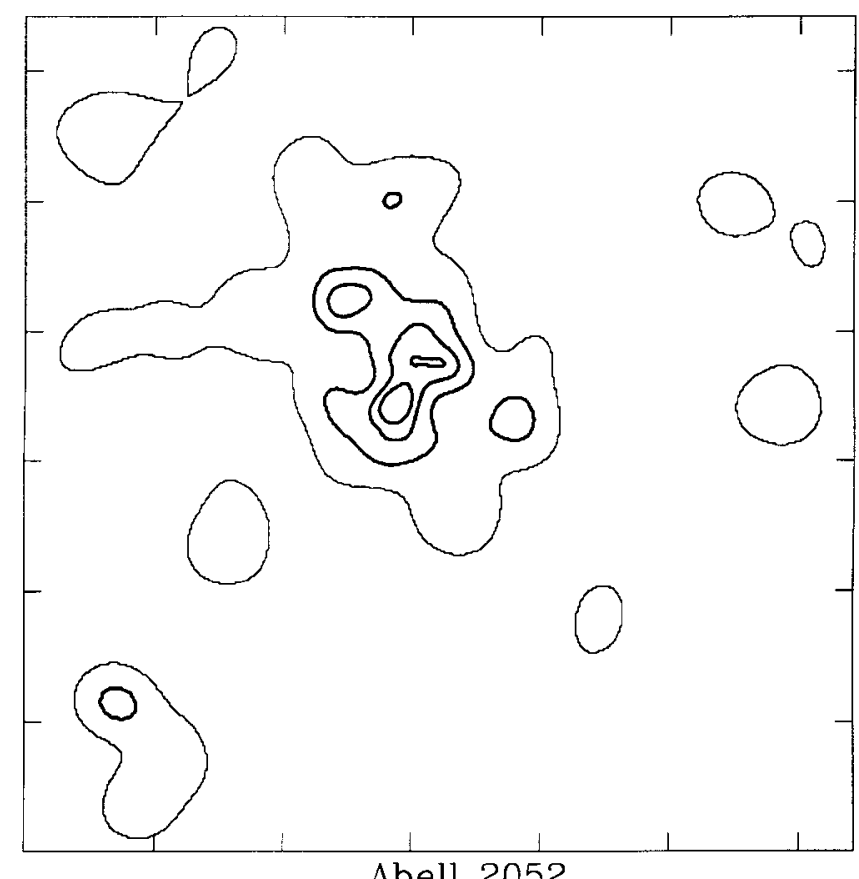

Abell 2052

Fig. 10. 


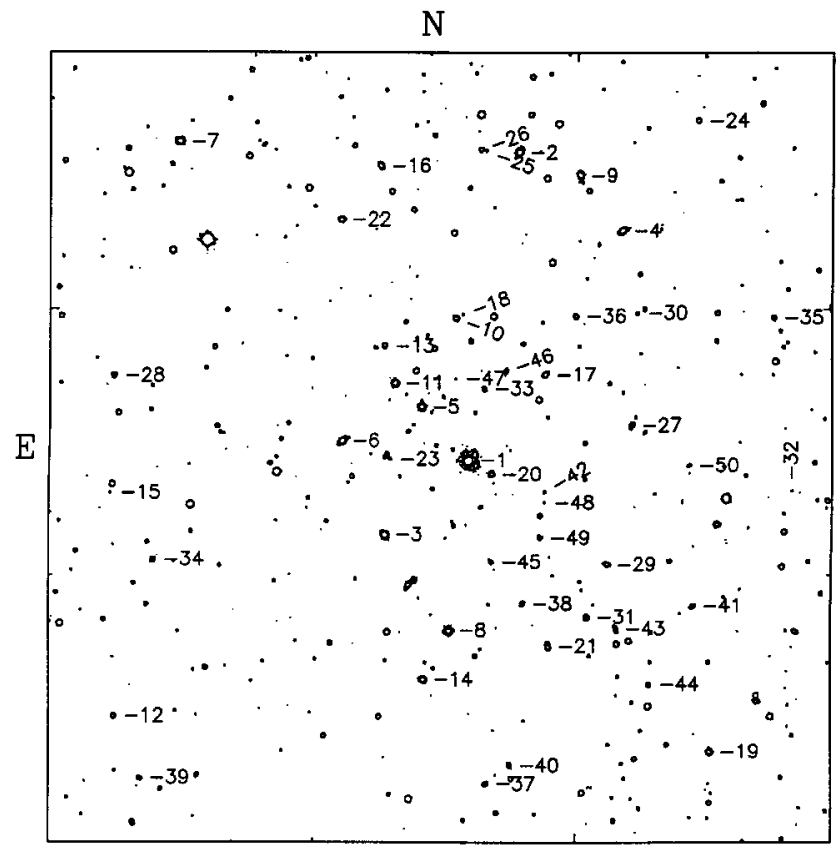

Abell 2063

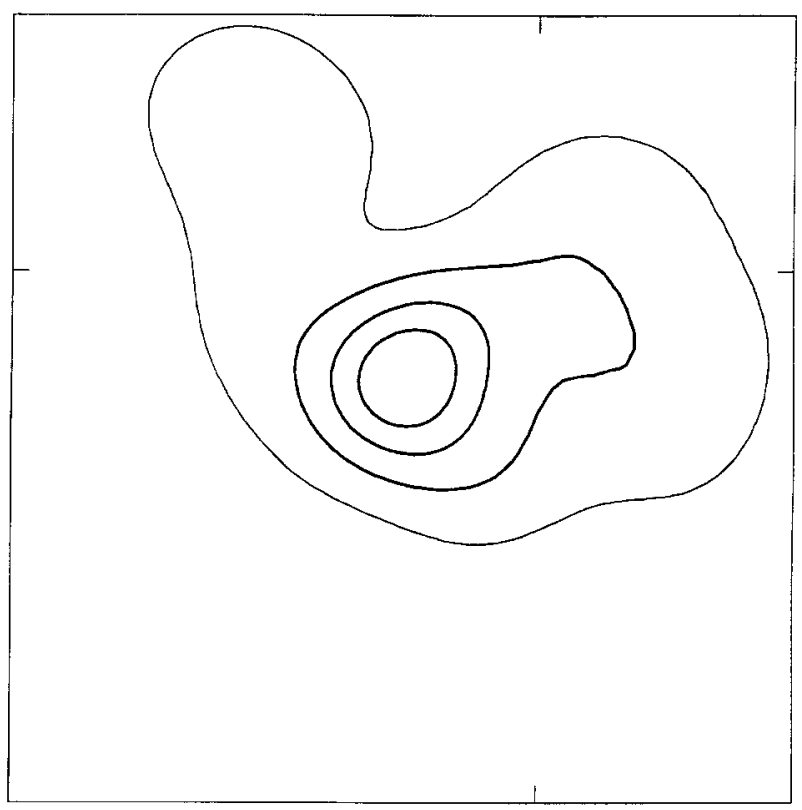

Abell 2063

Fig. 11.

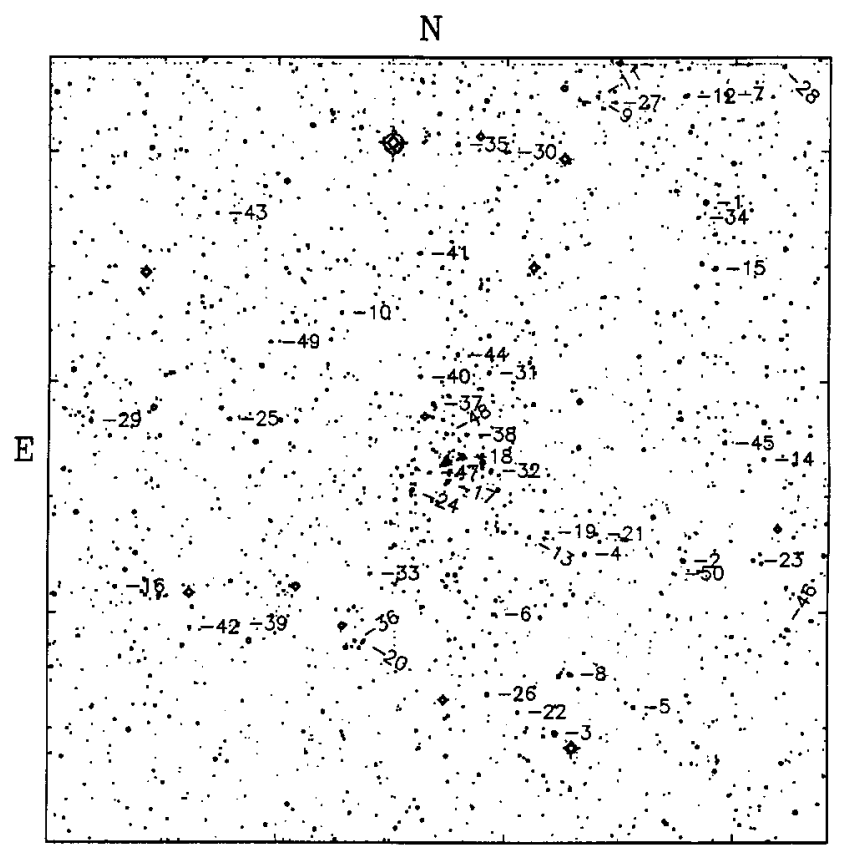

Abell 2065

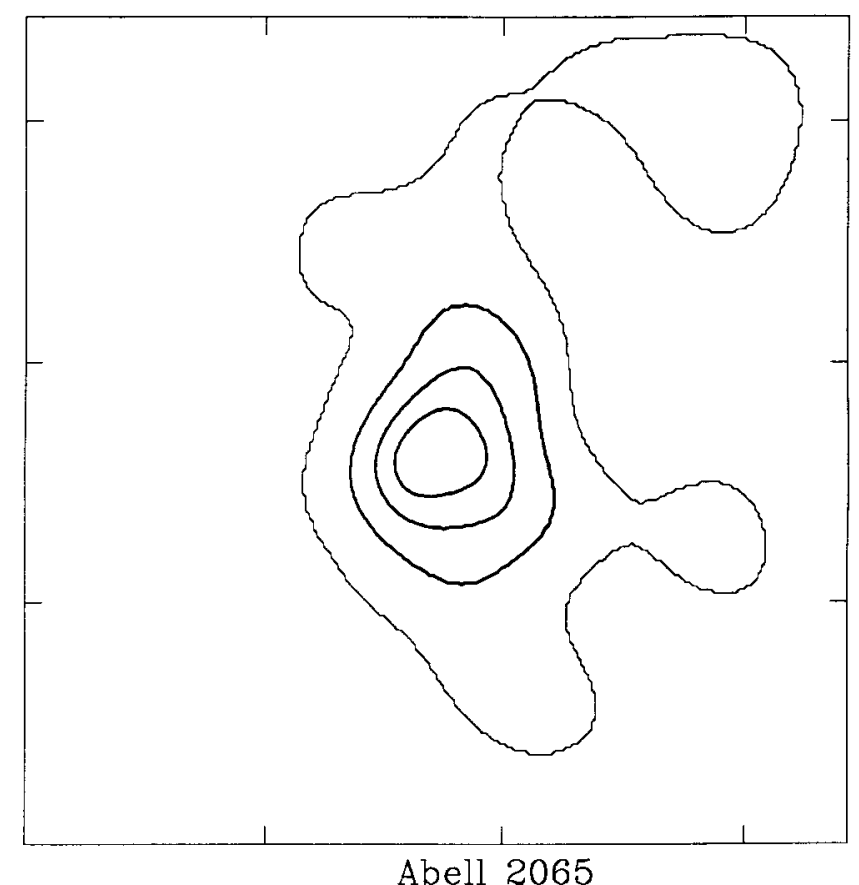

Abell 2065

Fig. 12. 


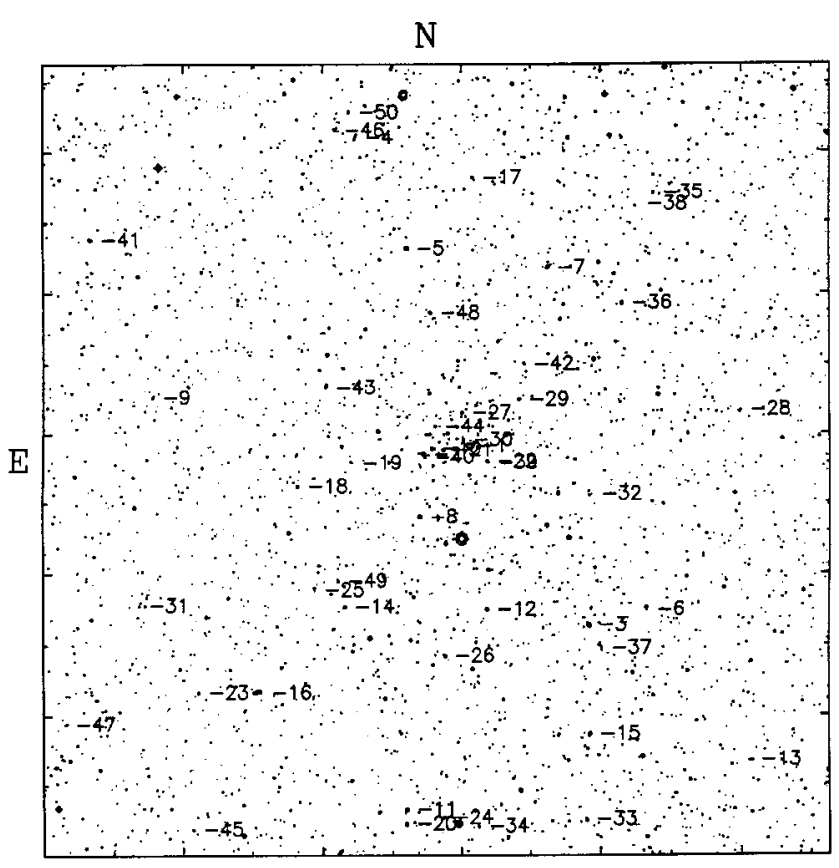

Abell 2593

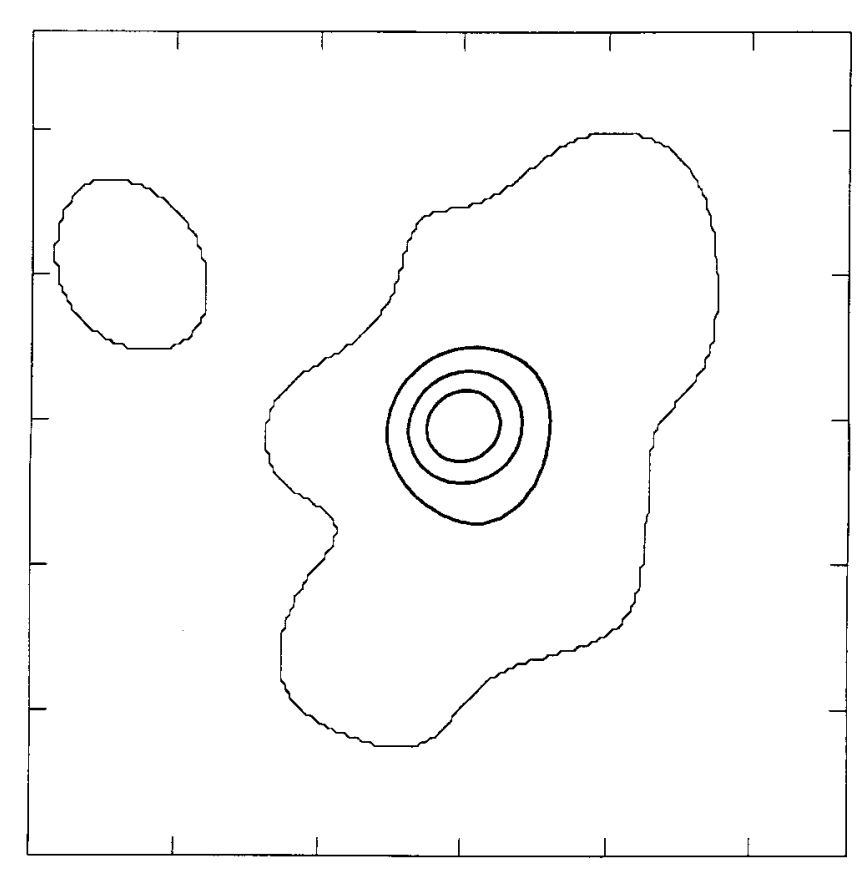

Abell 2593

Fig. 13.
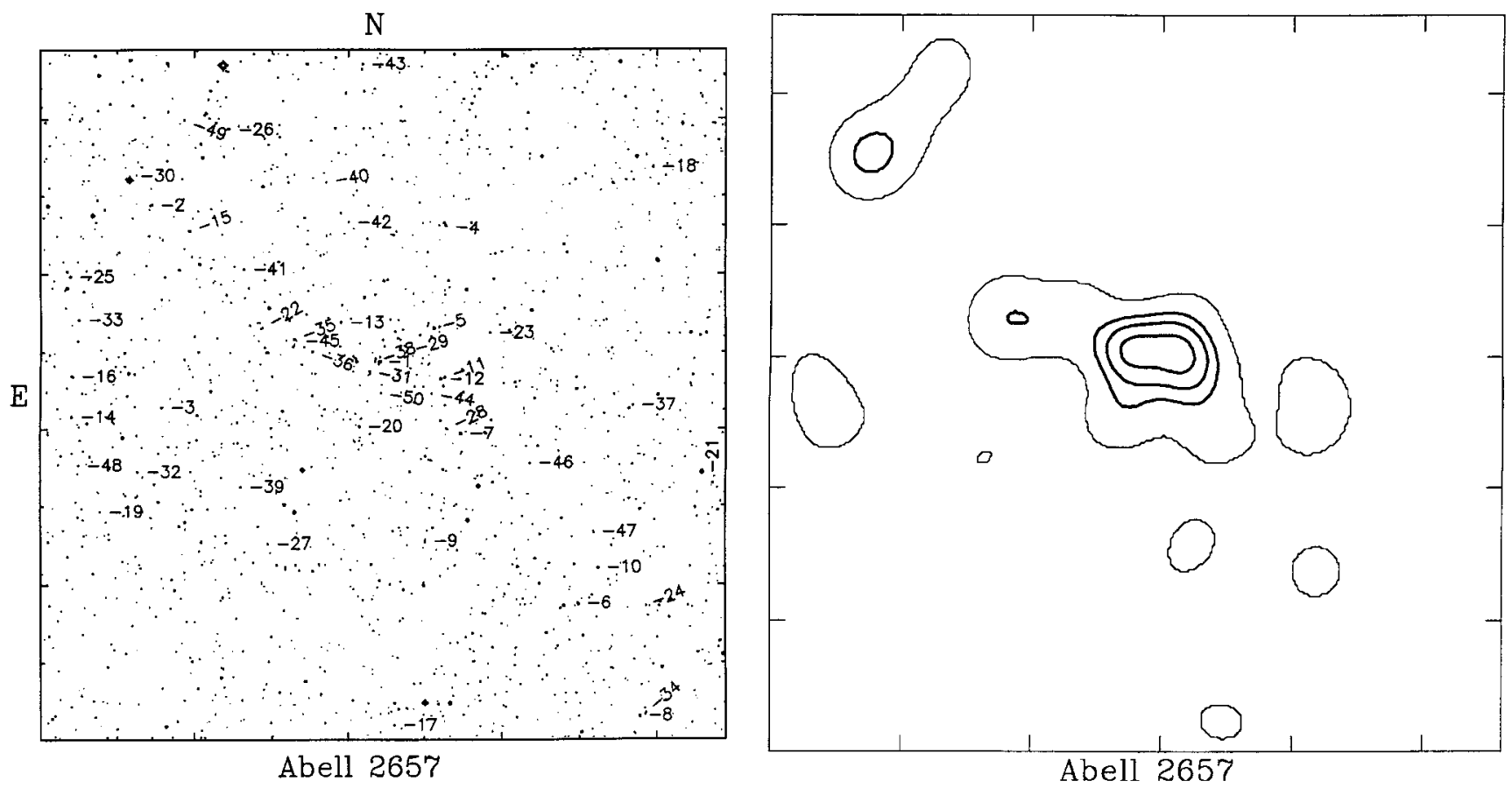

Fig. 14. 

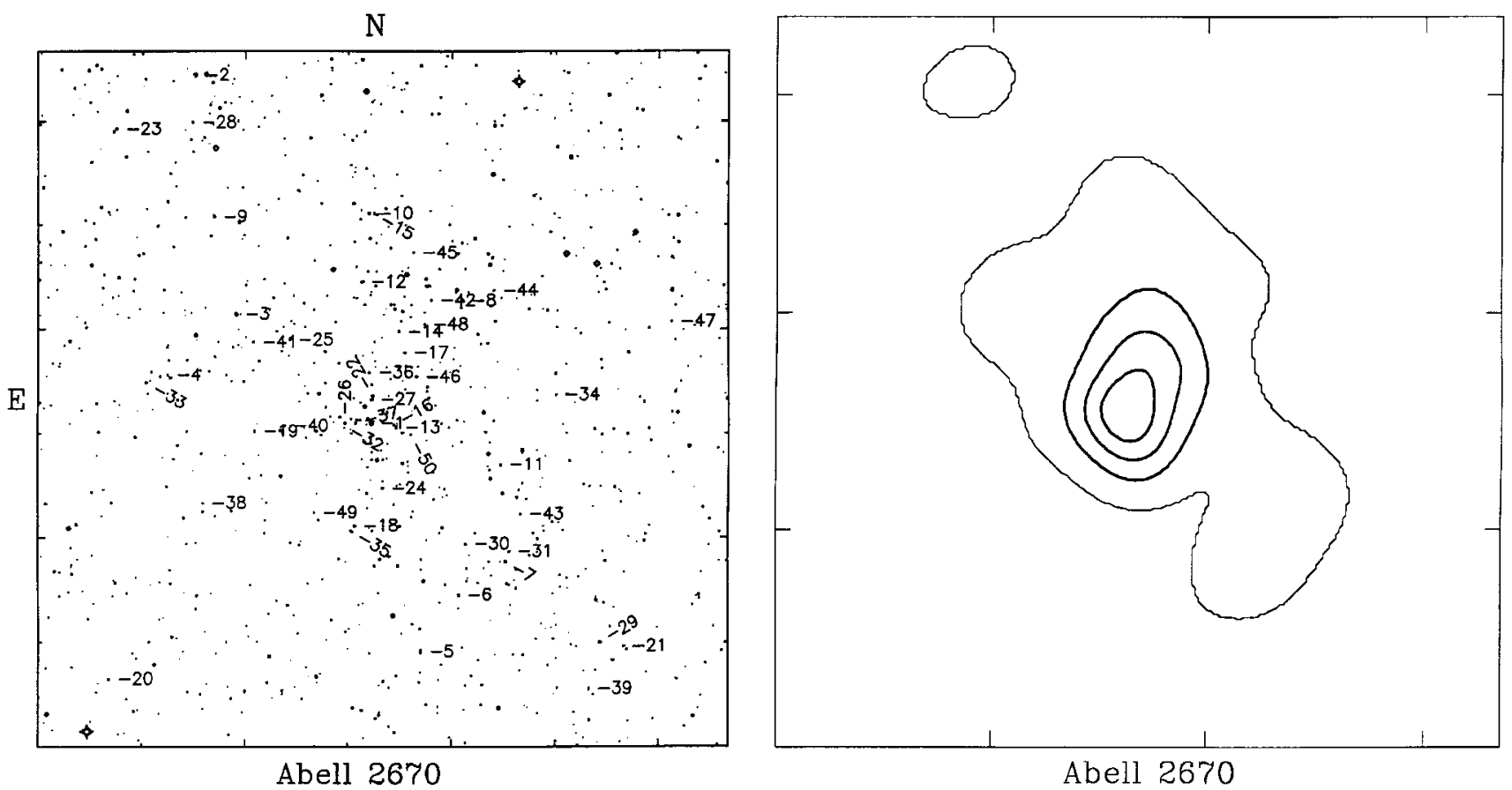

Fig. 15.

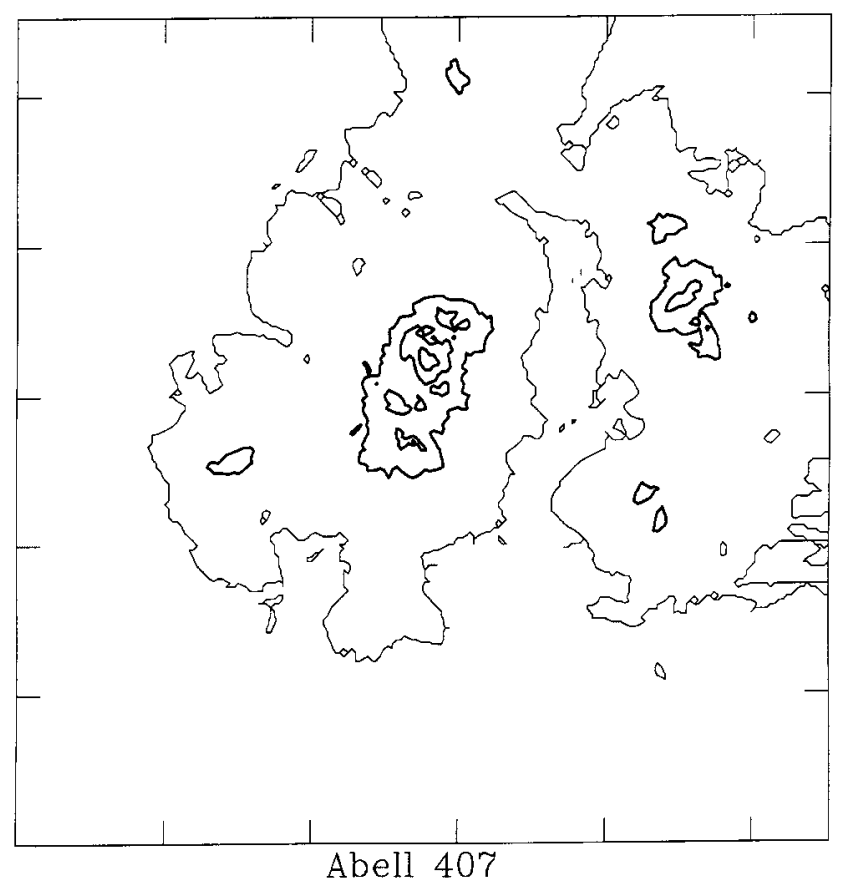

Fig. 16. Isodensity map of Abell 407 computed with the algorithm used in Trèvese et al. (1992), to be compared with 3b 